\title{
Image Fusion based on Local Pixel Information with Rough-Fuzzy C-means Approach
}

\author{
Rajvi Patel \\ Research Scholar \\ CE Department \\ Parul Institute of \\ Technology,Limda, \\ Vadodara, Gujarat.
}

\author{
Manali Rajput \\ Asst. Prof CSE Department \\ Parul Institute of \\ Technology,Limda, \\ Vadodara, Gujarat
}

\author{
Pramit Parekh \\ M.Tech, IT \\ Chandubhai S. Patel Institute of \\ Technology, Changa, \\ Anand, Gujarat.
}

\begin{abstract}
With the increased development of technology, it is necessary to retrieve information from multi source images in order to produce a high quality fused image with spatial and spectral information. Image Fusion is a process which allows the combination of the relevant information from a set of images into a single image where the resultant fused image will be more informative than any of the input images. Though the fused image can have complementary spatial and spectral resolution characteristics, the existing image fusion techniques can distort the spectral information of the multispectral data while merging. In this Paper, a rough set theory based fuzzy c-means approach is introduced for image fusion. The distribution of the local information and spatial constraint affect the damping extent of the pixels in neighbors. With the weighted rough and fuzzy factors depends on the space distance of all the neighboring pixels and their graylevel difference accurately measure the variance and enhance its robustness to noise and outliers.
\end{abstract}

\section{Keywords}

Multi-focus image fusion, RFCM.

\section{INTRODUCTION}

Usually, various images of the same scene can be obtained to enhance the robustness of image processing system. However, viewing and analyzing a series of images separately are not convenient and efficient. Image fusion is an effective technique to resolve this problem by combining complementary information from multiple images into a fused image, which is very useful for human or machine perception.

Recently, many image fusion algorithms have been developed to merge multi-focus images. In general, these algorithms can be classified into two groups: transform domain fusion and spatial domain fusion. Generally, traditional multi-focus image fusion methods can generate satisfactory results for source images captured in static scenes. However, few researchers studied the problem of multifocus image fusion in dynamic scenes with camera movement or object motion. In the dynamic scenes, the contents in the same position of multiple source images may be different. In those positions, the transform domain methods simply fuse these coefficients which represent more salient features to generate the fused image without taking into consideration that the features are probably derived from different contents. Thus, these methods usually suffer from artifacts in the fused image because of the inconsistence of image contents. For the majority of spatial domain methods, the focus information estimated by image variance, image gradient or spatial frequency is used to determine the focused pixel or region. However, in dynamic scenes, by using focus information alone, we cannot accurately decide whether a pixel or region is blurred or not. The reason is that the pixel or region in the same location of different source images may be comprised of different contents due to camera movement or object motion. Besides, traditional pixel based and region based methods cannot obtain very accurate fusion results when the patterns in the source images become complex.

In a novel multi-focus image fusion algorithm[1] with image matting which consists of the following three steps: first, morphological filtering is performed on each source image to measure focus. Then, the focus information is forwarded to image matting to find the focused object accurately. At last, the obtained focused objects of different source images are fused together to construct the fused image. The most important contribution of this approach is that the strong correlations between nearby pixels and the focus information of multi-focus images are combined together through image matting. Therefore, this algorithm can well resolve the problem of fusion of multi-focus images in dynamic scenes. Besides, another limitation of traditional spatial domain fusion methods is that their performances may degrade when image patterns become complex. On the contrary, since image matting is able to find very accurate outline of the focused object, our method can obtain very accurate fusion results in such situation. Experiments on various dynamic and static multi-focus image sets demonstrate that this method produces the state-of-the-art performance in generating satisfactory fused images, while traditional methods bring in different levels and types of undesirable artifacts.

The existing image fusion methodology suffers from the following problems:

1. The median filter is performed by taking the magnitude of all the vectors within a mask and sorting them which creates high computational cost.

2. The median filter is less effective in removing Gaussian or random-intensity noise. It removes noise only if the noisy pixels occupy less than one half of the neighborhood area which reduces the accuracy of the resultant image.

3. A threshold parameter needs to be tuned for image matting which requires underlying knowledge of the image intensity distribution.

To overcome these problem Rough fuzzy C-mean Approach is introduced. It improve the efficiency of the edges area of the object in the image and gives more qualitative output fused image. 


\section{RFCM (ROUGH FUZZY C-MEAN)}

The process begins by randomly choosing c objects as the centroids of the c clusters. The memberships are calculated based on the relative distance of the object $x_{j}$ to the centroids $\left\{\mathrm{v}_{\mathrm{i}}\right\}$. After computing the memberships of all the objects, the new centroids of the clusters are calculated. The process stops when the centroids stabilize. That is, the centroids from the previous iteration are identical to those generated in the current iteration.

Cluster analysis is a technique for finding natural groups present in the data. It divides a given data set into a set of clusters in such a way that two objects from the same cluster are as similar as possible and the objects from different clusters are as dissimilar as possible.

The membership function of fuzzy sets enables efficient handling of overlapping partitions, the concept of lower and upper approximations of rough sets deals with uncertainty, vagueness etc.

Image can be considered as an array of fuzzy singletons, after pre-filtering, each with a value of membership denoting the degree of brightness level according to membership function

The fundamental concept behind Rough Set Theory is the approximation of lower and upper spaces of a set, the approximation of spaces being the formal classification of knowledge regarding the interest domain. The subset generated by lower approximations is characterized by objects that will definitely form part of an interest subset, whereas the upper approximation is characterized by objects that will possibly form part of an interest subset. Every subset defined through upper and lower approximation is known as Rough Set. There are two types of approximations that are used in Rough Sets Theory.[13]

\section{a. Lower Approximation (B_low)}

Lower Approximation is a description of the domain objects that are known with certainty to belong to the subset of interest. The Lower Approximation Set of a set X, with regard to $\mathrm{R}$ is the set of all of objects, which certainly can be classified with $\mathrm{X}$ regarding $\mathrm{R}$, that is, set $\mathrm{B} \_$low.

$$
\text { B_low }=\bigcup\{Y \in U / B: Y \subseteq X\}
$$

\section{b. Upper Approximation (B_up)}

Upper Approximation is a description of the objects that possibly belong to the subset of interest. The Upper Approximation Set of a set $\mathrm{X}$ regarding $\mathrm{R}$ is the set of all of objects which can be possibly classified with $\mathrm{X}$ regarding $\mathrm{R}$, that is, set $\mathrm{B}$ _up.

$$
\text { B_up }=\bigcup\{Y \in U / B: Y \cap X \neq \phi\}
$$

\section{c. Boundary Region (BR)}

Boundary Region is description of the objects that of a set $\mathrm{X}$ regarding $\mathrm{R}$ is the set of all the objects, which cannot be classified neither as $\mathrm{X}$ nor $-\mathrm{X}$ regarding $\mathrm{R}$.

If the boundary region is a set $X=\varnothing$ (Empty), then the set is considered "Crisp", that is, exact in relation to R; otherwise, if the boundary region is a set $X \neq \varnothing$ (empty) the set $X$ "Rough" is considered. In that the boundary region is $B R=B \_u p-$ B_low.

\subsection{The rough set theory has the following characteristics:}

1. Each set is considered as an interval or rough set i.e. each interval/rough set is grouped, where lower and upper approximations B_low and B_up are characteristics of rough set X.

2. According to the elementary set theory, the data point should belong to at least one set.

a) An object $v$ can be part of at most one lower approximation. This implies that any two lower approximations do not overlap.

b) An object $v$ that is member of a lower approximation of a set is also part of its upper approximation $\left(v \in B_{-} l o w \rightarrow v \in B_{-} u p\right)$. This implies that a lower approximation of a set is a subset of its corresponding upper approximation $\left(B \_l o w \subseteq B \_u p\right)$.

c) If an object $v$ is not part of any lower approximation, it belongs to two or more upper approximations. This implies that an object cannot belong to only a single boundary region but not in upper approximation.

\section{PROPOSED ALGORITHM}

The proposed method is divided into three phases:

Phase 1: Pre-processing

The input image is preprocessed for the noise removal

Phase 2: Compression Phase

a. Initialization.

b. Compute fuzzy membership functions

c. Compute cluster centers

d. Assign samples to approximations.

e. Repeat steps a-d until convergence.

Phase 3: Evaluation Phase

The results obtained are compared on different measure

\subsubsection{Algorithm}

Input: Image

Output: Fusion Image

1. Preprocess the image

Resize the Image

Noise removal

2. The number of clusters is initialized.

3. Computer membership $u_{i k}$ for $\mathrm{C}$ clusters and $N$ data objects as

$$
u_{i k}=\frac{1}{\sum_{j=1}^{C}\left(\frac{d_{i k}}{d_{j k}}\right)^{\frac{2}{m-1}}}
$$


4. Let $u_{i k}$ be the maximum and $u_{j k}$ be the next to maximum

5. If $u_{i k}-u_{j k}$ is less than some threshold value

$$
\text { then } x_{k} \in \bar{B} U_{i} \text { and } x_{k} \in \bar{B} U_{j} \text { and } x_{k} \text { cannot }
$$

be a member of any lower approximation

else $x_{k} \in \underline{B U}_{i}$ such that membership $u_{i k}$ is maximum over the $C$ clusters

6. Compute the new mean for each cluster $U_{i}$ as

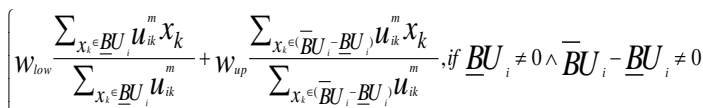

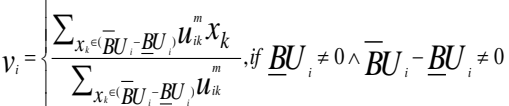

$$
\begin{aligned}
& \frac{\sum_{x_{k} \in \underline{B U}} u_{i k}^{m} x_{k}}{\sum_{x_{k} \in \underline{B U}} u_{i k}^{m}} \text {, otherwise }
\end{aligned}
$$

7. Repeat Steps 2 to Steps 5 until converge i.e. there are no more new assignments.

8. Obtain the fused Image as resultant

\subsubsection{Pre Processing:}

The input image is a two shared image that has been preprocessed with the application of the median filter. It is also useful in preserving edges in an image while reducing random noise. Impulsive or salt-and pepper noise can occur due to a random bit error in a communication channel. In a median filter, a window slides along the image, and the median intensity value of the pixels within the window becomes the output intensity of the pixel being processed. The preprocessed image is segmented for finding the proper edges of the image that is two-shared. The obtained segmented image is clustered with Rough-Fuzzy C Means approach for finding the final image from the two shared images that has noise in both the half share of the images. The resultant image obtained is evaluated with the different measure for analyzing the performance of the algorithm with the conventional one.

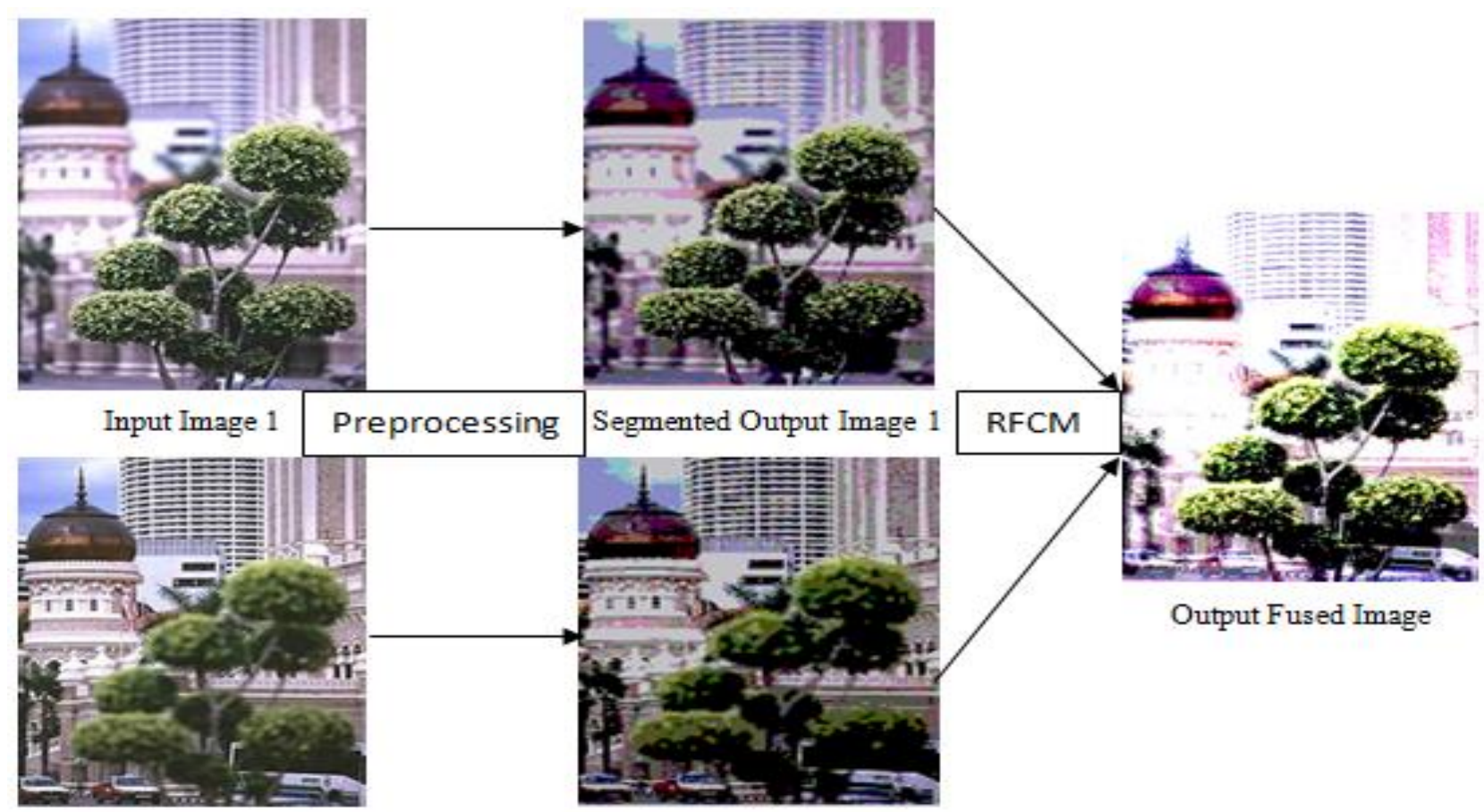

Input Image 2

Segmented Output Image 2

Fig 1 .Image Fusion Based on Rough Fuzzy C-Mean Approach 
There are Two input Images. As Shown Fig 1, first input image of city, Tree is focused which is in foreground of image and in second input image of city, Building is focused which is in back ground of image. The unfocused region is blurred. Image will be resized and noise will be removed in preprocessing step. Then the segmented output of input images is generated. In these Step focused region is measured. After that final output of fused image is generated which is more qualitable than input images.

\section{EXPERIMENTAL ANALYSIS AND RESULTS}

\subsection{Quality Matrix}

Quantitative metrics used in the analysis of the proposed system are as following:

\section{Overall Cross Entropy (OCE):}

It gives the difference between the input images and the fused image. Lower the value better is the fusion results obtained. It is given as:

$$
\operatorname{OCE}\left(I_{A}, I_{B}, F\right)=\left(C E\left(I_{A}, F\right)+C E\left(I_{B}, F\right)\right) / 2
$$

Where, IA and IB are the input images of different modality, $F$ is the fused image, $\mathrm{CE}\left(\mathrm{I}_{\mathrm{A}}, \mathrm{F}\right)$ and $\mathrm{CE}\left(\mathrm{I}_{\mathrm{B}}, \mathrm{F}\right)$ is the cross entropy of the input images with the fused image.

\section{Peak Signal to Noise Ratio (PSNR)}

PSNR is used to measure the quality of the image with respect to the original input image. It is defined as given below:

$$
\operatorname{MSE}=1 / \mathrm{pq} \Sigma_{\mathrm{i}=0}^{\mathrm{p}-1} \Sigma_{\mathrm{j}=0}^{\mathrm{q}-1}[\mathrm{~A}(\mathrm{i}, \mathrm{j})-\mathrm{B}(\mathrm{i}, \mathrm{j})]^{2}
$$

$$
\mathrm{PSNR}=10 \log _{10}\left(\mathrm{MAX}^{2} / \mathrm{MSE}\right)
$$

Where, MAX is the maximum value in an image. $p, q$ are the height and weight of an image. $A(i, j)$ is the value of input image and $\mathrm{B}(\mathrm{i}, \mathrm{j})$ is the value of fused image.

\section{Signal to Noise Ratio (SNR)}

It is defined as the ratio of mean pixel value to that of standard deviation of the corresponding pixel values.

$$
\mathrm{SNR}=\text { Mean } / \text { Standard Deviation }
$$

It gives the contrast information of an image. Higher value indicates more contrast.

\section{Structural Similarity Index (SSIM)}

It gives the association between the structural information changes within the images and the perceived distortion of the images. It is defined as a measure to assess similarity between two images $\mathrm{A}$ and $\mathrm{B}$ by the expression

$$
\begin{gathered}
\operatorname{SSIM}(\mathrm{A}, \mathrm{B})=\left(2\left(\mu_{\mathrm{A}} \mu_{\mathrm{B}}+\mathrm{k} 1\right) * 2\left(\sigma_{\mathrm{AB}}+\mathrm{k} 2\right)\right) / \\
\left(\left(\mu_{\mathrm{A}}^{2}+\mu_{\mathrm{B}}^{2}+\mathrm{k} 1\right) *\left(\sigma \mathrm{A} 2+\sigma_{\mathrm{B}}{ }^{2}+\mathrm{k} 2\right)\right)
\end{gathered}
$$

Where, $\mu_{\mathrm{A}}$ and $\mu_{\mathrm{B}}$ denotes the mean intensities, $\sigma_{\mathrm{A}}$ and $\sigma_{\mathrm{B}}$ denote the standard deviation, $\sigma_{\mathrm{AB}}$ gives the covariance of $\mathrm{A}$ and $\mathrm{B}, \mathrm{k} 1$ and $\mathrm{k} 2$ are constants.

\subsection{Experimental Setup}

For the proposed method, the structure element B used for two types of top-hat transforms is defined as $5 \times 5$ diamond matrix. The median filtering is performed on an $8 \times 8$ neighborhood. When the number of pixels in the neighborhood is even, the median value is calculated by choosing the bigger one of the two medians.
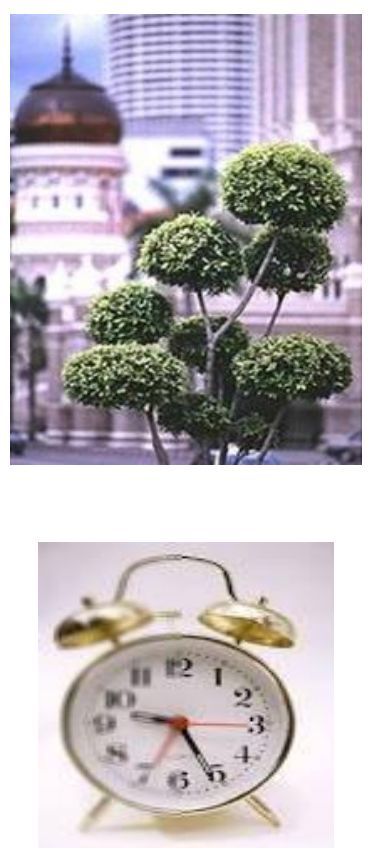

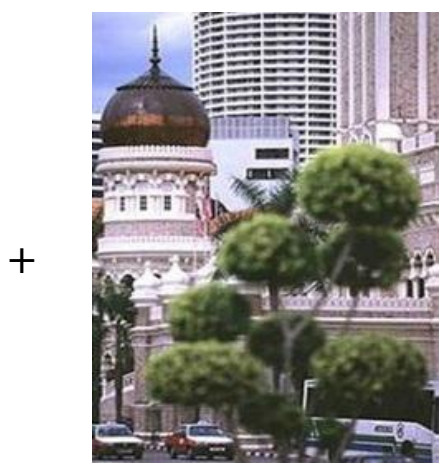

Fig 1. Experiment 1

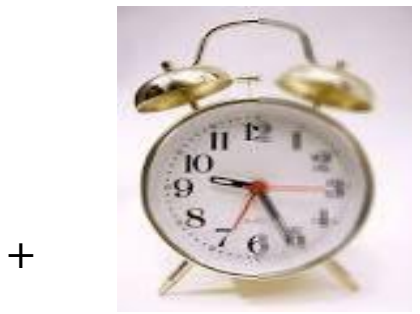

Fig 2. Experiment 2
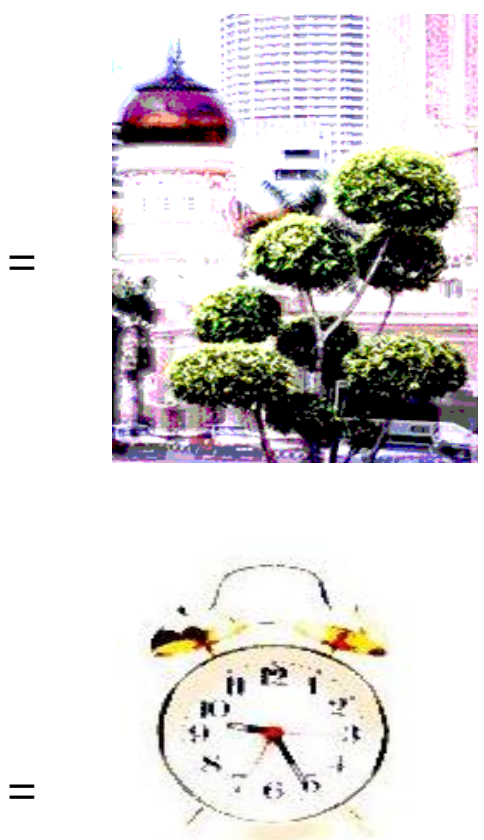

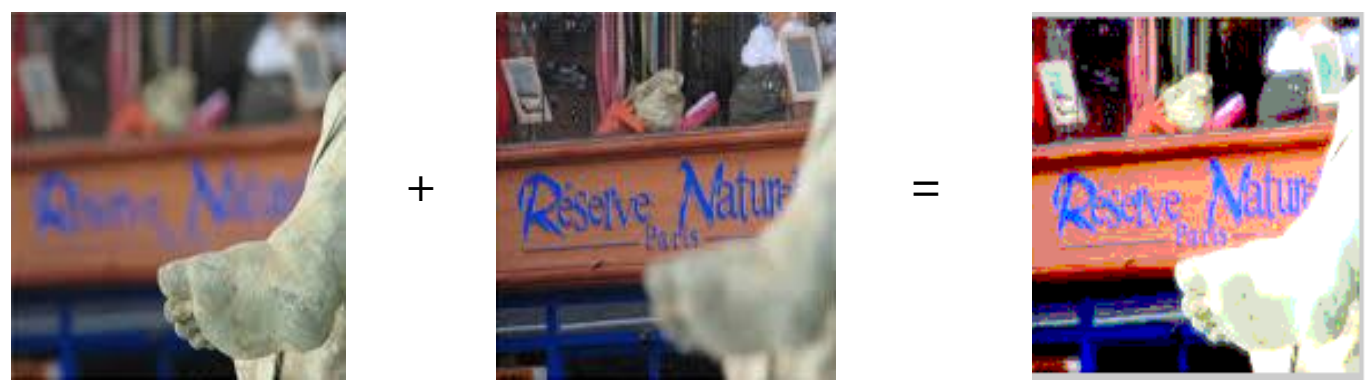

Fig 3. Experiment 3

\begin{tabular}{|c|c|c|c|c|c|c|c|}
\hline Metrics & Methods & \multicolumn{2}{|c|}{ Experiment1 } & \multicolumn{2}{|c|}{ Experiment 2} & \multicolumn{2}{|c|}{ Experiment 3} \\
\hline \multirow{4}{*}{ PSNR } & Averaging & 29.6933 & 30.4139 & 28.5705 & 28.5635 & 33.4035 & 32.9279 \\
\hline & Maximum & 29.9326 & 30.4797 & 29.4772 & 29.4636 & 35.6042 & 35.0879 \\
\hline & Existing & 30.6048 & 31.1900 & 30.5639 & 30.2389 & 35.4382 & 35.8765 \\
\hline & Proposed & 31.3212 & 32.5138 & 31.2509 & 31.2431 & 35.4855 & 35.6765 \\
\hline \multirow{4}{*}{ SNR } & Averaging & \multirow{4}{*}{\multicolumn{2}{|c|}{$\begin{array}{l}3.2181 \\
2.2886 \\
2.2476 \\
1.6688\end{array}$}} & \multirow{4}{*}{\multicolumn{2}{|c|}{$\begin{array}{l}9.7278 \\
5.0906 \\
4.9830 \\
4.1959\end{array}$}} & \multirow{4}{*}{\multicolumn{2}{|c|}{$\begin{array}{l}2.4665 \\
2.0631 \\
1.4980 \\
1.5057\end{array}$}} \\
\hline & Maximum & & & & & & \\
\hline & Existing & & & & & & \\
\hline & Proposed & & & & & & \\
\hline \multirow{4}{*}{ SSIM } & Averaging & 100 & 100 & 100 & 100 & 99.9601 & 99.9589 \\
\hline & Maximum & 100 & 100 & 100 & 100 & 99.9601 & 99.9589 \\
\hline & Existing & 75.6128 & 76.1014 & 95.6546 & 95.7849 & 87.8575 & 87.7489 \\
\hline & Proposed & 65.3642 & 65.3643 & 82.8127 & 82.8127 & 77.8944 & 77.9436 \\
\hline \multirow{4}{*}{ Entropy } & Averaging & \multirow{4}{*}{\multicolumn{2}{|c|}{$\begin{array}{l}4.0269 \\
7.7311 \\
3.5164 \\
3.1638\end{array}$}} & \multirow{4}{*}{\multicolumn{2}{|c|}{$\begin{array}{l}0.9115 \\
6.9792 \\
2.8049 \\
1.1560\end{array}$}} & \multirow{4}{*}{\multicolumn{2}{|c|}{$\begin{array}{l}5.3980 \\
7.7218 \\
4.6754 \\
3.5273\end{array}$}} \\
\hline & Maximum & & & & & & \\
\hline & Existing & & & & & & \\
\hline & Proposed & & & & & & \\
\hline \multirow{4}{*}{ MSE } & Averaging & 4.8697 & 3.4945 & 8.1669 & 8.1933 & 1.0092 & 1.0980 \\
\hline & Maximum & 4.9873 & 3.3901 & 8.06321 & 8.0648 & 3.4689 & 2.872 \\
\hline & Existing & 2.5438 & 2.3874 & 2.2345 & 2.1983 & 3.3700 & 2.2142 \\
\hline & Proposed & 2.3011 & 2.4041 & 2.3767 & 2.3853 & 3.3812 & 2.0965 \\
\hline
\end{tabular}

Table 1: Comparative Analysis of Quality Matrix

\section{CONCLUSION}

Image fusion can be broadly defined as the process of combing multiple input images or some of their features into a single image without the introduction of distortion or loss of information. A rough set theory based fuzzy c-means approach is introduced for image fusion. The distribution of the local information and spatial constraint affect the damping extent of the pixels in neighbors. With the weighted rough and fuzzy factors depends on the space distance of all the neighboring pixels and their gray-level difference accurately measure the variance and enhance its robustness to noise and outliers.

\section{ACKNOWLEDGMENTS}

The authors would like to thank the editor and anonymous reviewers for their detailed review. And also thank to family and friends for their support.

\section{REFERENCES}

[1] Shutao Li, Xudong Kang, Jianwen Hu, Bin Yang, "Image matting for fusion of multi-focus images in dynamic scenes", Journal of Information Fusion, pp. 147$162,2013$.

[2] Shen, Irene Cheng, and Anup Basu, "Cross-Scale Coefficient Selection for Volumetric Medical Image Fusion", IEEE Transactions on Biomedical Engineering, Vol. 60, No. 4, pp. 1-10, 2013.

[3] Xiao Xiang Zhu and Richard Bamler, "A Sparse Image Fusion Algorithm With Application to Pan-Sharpening", IEEE Transaction on Geoscience and Remote Sensing, Vol. 51, No. 5, pp.2827-2836, 2013.

[4] Andreas Ellmauthaler, CarlaL.Pagliari and Eduardo A. B. da Silva, "Multiscale Image Fusion Using the Undecimated Wavelet Transform With Spectral Factorization and Nonorthogonal Filter Banks", IEEE Transaction in Image Processing, Vol. 22, No.3,pp.10051017, 2013 
[5] Vivek Angoth, CYN Dwith and Amarjot Singh, "A Novel Wavelet Based Image Fusion for Brain Tumor Detection", International Journal of Computer Vision and Signal Processing, pp. 1-7, 2013.

[6] S. A. Quadri and Othman Sidek, "Pixel-Level Image Fusion using Kalman Algorithm", International Journal of Signal Processing, Image Processing and Pattern Recognition, Vol. 6,pp.75-86 2013.

[7] K. Venkateswaran, N. Kasthuri and Arathy C. Haran V., "Unsupervised Change Detection using Image Fusion and Kernel K-Means Clustering", International Conference on Innovations In Intelligent Instrumentation, Optimization And Signal Processing,pp.1-5, 2013.

[8] Joan Duran, Antoni Buades, Bartomeu Coll and Catalina Sbert, "Implementation of Nonlocal Pansharpening Image Fusion", SIAM Journal on Imaging Sciences, 2014.

[9] Pramit Parekh, Nehal Patel, Robinson Macwan, Pritesh Kumar Prajapati, Sarita Visavalia, "Comparative Study and Analysis of Medical Image Fusion Techniques"(0975-8887)Volume.90-No.19,pp.-1216,March(2014).

[10] Prof. Keyur N. Brahmbhatt, Dr. Ramji M. Makwana, "Comparative study on image fusion methods in spatial domain", International journal of advanced research in engineering and technology (IJARET) (2013).

[11] Mr. Rajenda Pandit Desale, Prof. Sarita V. Verma, "Study and Analysis of PCA, DCT \& DWT based Image Fusion Techniques", International Conference on Signal Processing, Image Processing and Pattern Recognition [ICSIPR] (2013).

[12] KavitaTewari, Leena Shah, "Pixel Level Image Fusion Based on Spatial and Transform Domain", International Conference on Computer Science, Information and Technology, Pune, ISBN-978-93-81693-83-4 (2012)

[13] Zhengho Shi, yuyan Chao, Lifeng He, Tsuyoshi Nakamura and Hidenori Itoh "Rough Set Based FCM Algorithm for Image Segmentation" International Journal of Computational Science, 2007, vol-1, No. 1, pp58-68.

[14] Zexuan Ji, Quansen Suna, Yong Xia, Qiang Chena, Deshen Xia, Dagan Feng "Generalized rough fuzzy $c$ means algorithm for brain MR image segmentation" Computer Methods and Programs in Biomedicine 108(2012) 644-655

[15] Neelam Kumari, Bhawna Sharma, Dr. Deepti Gaur "Implementation of Possibilistic Fuzzy C-Means Clustering Algorithm in Matlab" International Journal of Scientific \& Engineering Research, Volume 3, Issue 11,pp-1-9, November-2012 ISSN 2229-5518 